\title{
Direct Yaw-Moment Control of 4-Wheel-Independent-Drive Electric Vehicle with Network-Induced Delays through Data-reduction Techniques
}

\author{
Shuju Wang ${ }^{1, a,},{ }^{*}$, Yingji Liu ${ }^{2}$ \\ ${ }^{1}$ Liaoning Technical University, Fuxin Liaoning123000, China \\ ${ }^{2}$ Research Institute of Highway, Ministry of Transport, Beijing 100088, China \\ awangshuju163@163.com
}

Keywords: Four-wheel-independent-drive electric vehicle, Controller Area Network, Networkinduced delay, Direct yaw-moment control, Data-reduction.

Abstract: Direct yaw-moment control (DYC) has obvious advantages in centralized-drive vehicles, but in 4WID-EV networked control system, the network-induced delays would reduce control performance of DYC and even deteriorate the 4WID-EV networked control system. To ensure robustness of DYC and reduce the adverse impact of message time-delays, an alternative option is select to reduce the data transforming in network, and a mended data-reduction algorithm is proposed in this paper, based on assembling the four motor control messages into one message. The results of co-simulation with Simulink and CarSim demonstrate the effectiveness of the proposed algorithm.

\section{Introduction}

4-wheel-independent-drive electric vehicle(4WID-EV) is becoming increasingly popular in academia and industry in recent years[1-3]. Advanced electric motors with accurate and quick torque generations, which ensure 4WID-EV have obvious advantages in terms of direct yaw-moment control (DYC) through flexible differential driving functions[4]. It is well known that the in-vehicle network such as controller area network (CAN) have considerable advantages over the traditional point-to-point communication. However, in-vehicle network would also induce the probability of time varying delays, which would degrade control performance or even deteriorate the system.

To deal with in-vehicle network delays, [5] presented an $\mathrm{H} \infty$-based delay-tolerant linear quadratic regulator control method. To ensure robustness of DYC and deal with network-induced delays, [6] presented a parameter-dependent fuzzy sliding mode control method based on the real-time information of vehicle states and delays. To reduce the adverse impact of message time-delays in networked control systems, varieties of methods have been developed to maximally utilize the limited network bandwidth, and message scheduling is a very important one. in this paper, an alternative option is proposed by introducing the data-reduction (DR) techniques in higher layer protocol. DR is used to reduce the amount of data to be transferred. Its goal is to communicate the same amount of information using less bus bandwidth, and reduce adverse impact of networkinduced time delays, it would be acceptable, provided that it does not increase the message latencies significantly so that the safety of the vehicle is not compromised.

\section{Control-Oriented Vehicle Lateral Dynamics Model.}

The DYC function is implemented by 4WID-EV controller, which is usually designed as a hierarchical controller including reference state model, motion controller unit, torque distribution unit, and estimation and processing unit. The reference state model is used to solve the reference states such as the reference sideslip angle $\beta_{\text {res }}$ and the reference yaw rate $\gamma_{\text {res }}$ according to vehicle 
speed $V$ and the front wheel steering angle $\delta$ from the driver. The reference states indicate the desired motion state by the driver. The motion controller unit is used to calculate the external yaw-moment $\Delta M_{\mathrm{Z}}$ to keep the sideslip angle $\beta$ and the yaw rate $\gamma$ tracking the reference states. The torque distribution unit is used to solve the longitude forces for 4 motors according to the $\Delta M_{\mathrm{Z}}$. The estimation and processing unit is used to measure or estimate states such as $V, \beta, \gamma$.

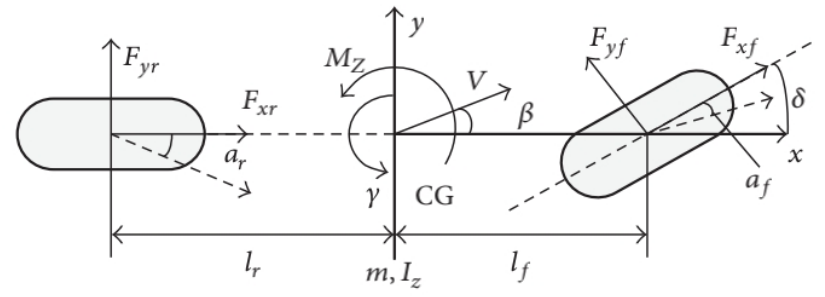

Fig. 1: 2-DOF model of control-oriented vehicle lateral dynamics.

As shown in Figure 1, a two-degree-of-freedom (2-DOF) vehicle model is used in the paper, where CG is the center of gravity; $m$ is the vehicle mass; $I_{\mathrm{z}}$ is the vehicle yaw inertia; $M_{\mathrm{z}}$ is the yaw moment; $F_{\mathrm{xf}}$ and $F_{\mathrm{xr}}$ are the longitude tire forces of front and rear wheels, respectively; $F_{\mathrm{yf}}$ and $F_{\mathrm{yr}}$ are the lateral tire forces of front and rear wheels, respectively; $\alpha_{\mathrm{f}}$ and $\alpha_{\mathrm{r}}$ are the slip angle of front and rear wheels, respectively. With the 2-DOF vehicle model, the state space formulation of controloriented vehicle lateral dynamics model for DYC of 4WID-EV is expressed as follows [6-8]:

$$
\stackrel{\mathrm{g}}{x}=A x+B u+E \delta
$$

\section{( 1 )}

Where

$$
x=[\beta, \gamma]^{T}, u=\Delta M_{z}, A=\left[\begin{array}{ll}
-2 \frac{c_{f}+c_{r}}{m V} & -2 \frac{c_{f} l_{f}-c_{r} l_{r}}{I_{z}}-1 \\
-2 \frac{c_{f} l_{f}-c_{r} l_{r}}{I_{z}} & -2 \frac{c_{f} l_{f}^{2}-c_{r} l_{r}^{2}}{I_{z} V}
\end{array}\right], B=\left[\begin{array}{l}
0 \\
\frac{1}{I_{z}}
\end{array}\right], E=\left[\begin{array}{l}
\frac{2 c_{f}}{m V} \\
\frac{2 c_{f} l_{f}}{I_{z}}
\end{array}\right] .
$$

$C_{\mathrm{r}}$ and $c_{\mathrm{f}}$ are the cornering stiffness of the rear and front tires, respectively.

In vehicle lateral motion control, the desired sideslip angle is generally selected to be zero to ensure vehicle stability, while the desired yaw rate is usually defined to ensure good handling performance. A widespread expression of the desired yaw rate is described as[6-8]:

$$
\mathrm{r}=R \delta
$$

where

$$
\mathrm{r}=\left[\begin{array}{c}
\beta_{\text {res }} \\
\gamma_{\text {res }}
\end{array}\right], \quad R=\left[0 \frac{V}{l_{f}+l_{r}+m V^{2}\left(c_{r} l_{r}-c_{f} l_{f}\right) / 2 c_{f} c_{r}\left(l_{f}+l_{r}\right)}\right]^{T} .
$$

In this paper, only the case that the network induced time delays are less than the control cycle period $T_{\mathrm{s}}$, which commonly occurs in most networked control systems, in the network control system (NCS) of DYC for 4WID-EV, the sensor node periodically samples the vehicle states with fixed period $T_{\mathrm{s}}$, the controller node and the actuator node operate in event-driven mode which means a task will be immediately implemented once a message arrives via CAN, and the task implementation time in each node is ignored. Without considering network-induced delays, the NCS of DYC for 4WID-EV runs like an ideal centralized control system with fixed sampling period . The controloriented discrete time model of the vehicle lateral dynamics along with reference state model can be written as [7]: 


$$
\left\{\begin{array}{l}
x_{k+1}=A_{d} x_{k}+B_{d} u_{k}+E_{d} \delta_{k} \\
r_{k}=R_{d} \delta_{k}
\end{array}\right.
$$

Where

$$
A_{d}=e^{A T_{s}}, R_{d}=R, B_{d}=\int_{0}^{T_{s}} e^{A\left(T_{s}-\theta\right)} d \theta \cdot B, E_{d}=\int_{0}^{T_{s}} e^{A\left(T_{s}-\theta\right)} d \theta \cdot E .
$$

With $x_{\mathrm{k}}, \delta_{\mathrm{k}}, u_{\mathrm{k}}, r_{\mathrm{k}}$ indicating the state vector, steering angle vector, control input vector, and the reference state vector at time $k T_{\mathrm{S}}$ respectively.

By defining $\Psi_{\mathrm{k}}$, which stands for the components caused by the message time delays, the controloriented discrete time model of the vehicle lateral dynamics with network-induced delays can be expressed as follows [7]:

$$
x_{k+1}=A_{d} x_{k}+B_{d} u_{k}+E_{d} \delta_{k}+\psi_{k}
$$

In order to mitigate the adverse impact caused by the network $\square$ induced time delays and simultaneously utilize the limited bandwidth, the mismatch term $\Psi_{\mathrm{k}}$ should be Minimized.

\section{Controller Design with data-reduction algorithm}

As indicated in [6,7], there are 4 messages containing control commands from the vehicle controller to four motor controller respectively, which will obviously cause the network delays, in order to mitigate the adverse impact caused by the network delays and utilize the limited bandwidth, we assemble the 4 control messages for four motor controller into one message using eight bytes data, two bytes data that is 16 bits will be enough to represent the torque for any motor in vehicle.

When vehicle working in stable state, most of the data bytes in control and command information frame sent by the central controller will does not change, which meet the conditions of implementation of data reduction. In order to mitigate the adverse impact caused by the network delays and utilize the limited bandwidth, this section put forward a MDR (mended data-reduction) algorithm, through scheduling CAN identifier, the algorithm achieve CAN network data compression in 4WID-EV .

In this paper, the ID design of network message using standard message format, the lowest bit ID.0 is set to DCB to represent data compression, the rest ten bits of the ID is used for data definition and information filtering. When initial message and messages without data compression are sent, set the $\mathrm{DCB}=0$, and if messages after data compression processing are sent, the DCB bit is 1 . In the MDR algorithm, the structure of the message frame is planned as in figure 2.

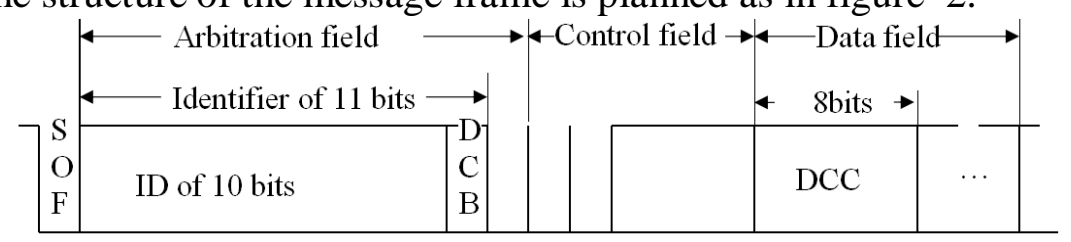

Fig. 2 The configuration of CAN data frame in MDR

Set the first byte of the data field to be the DCC field, by setting the $\mathrm{i}$ bit in DCC to indicate the $\mathrm{i}$ byte data in current message is exactly the same with backup information, the $\mathrm{i}$ data byte so there is no need to send for a change. The changed data bytes are sent behind the DCC field according to the original sequence. For example, if the first seven bytes of 8 bytes data do not change, the DCC code is shown in figure 3. 
Initial message data

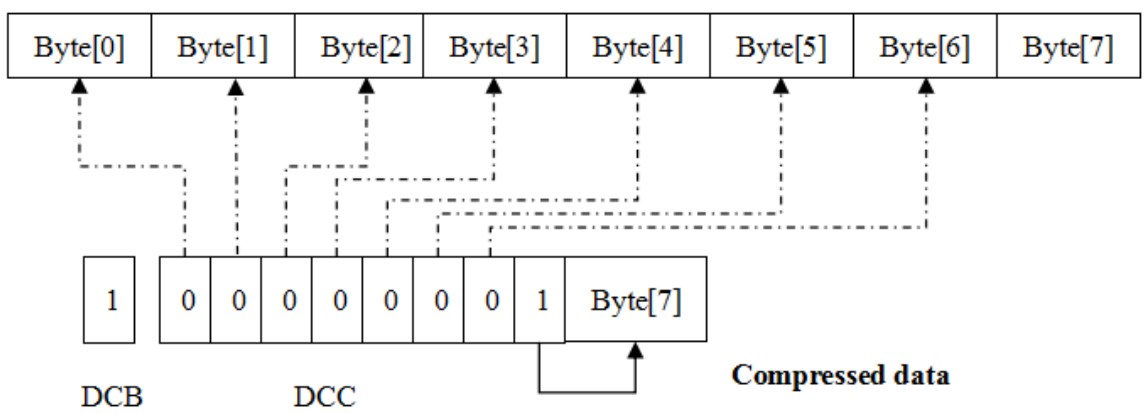

Fig.3 The configuration of CAN data frame in MDR

The core idea of data compression algorithm [9] is to reduce the data transmission. When the new message compared with the previous message, if some data content did not change, one bit can be used to represent a byte of data by setting corresponding bit in data compression encoding (DCC), DCC field in MDR algorithm occupied a byte of data, so only when the new information has more than one byte of data changed compared to the backup, the data compression technology MDR can be meaningful. When the vehicle controller produce a m message in the first time, it save a copy of the data in local as $m$ data backup, when $m$ come again, the controller will compare the new data with backup data, if there are two or more bytes of data does not change, then adopt the MDR, in order to make the receiving node can discriminate which byte in data was compressed, the DCB in sending message identifier is set to "1", and gives a DCC, the value of "1" in DCC said the byte of data is repeat, a value of " 0 " means no repeat in the byte. The encoding processing of the MDR algorithm is shown in figure 4.

Data decompression processing is to reconstruct the received information, each motor controller node keeps the backup information recently received, when receiving new information, the receiving node will judge the received data frame DCB firstly, if DCB is "1", then the received information frame is compressed, in this case the receiving unit put the receiving first byte of data field as compression codes, and use the rest bytes data and backup to reconstruct the whole data contents, MDR decompression processing is shown as in figure 5.

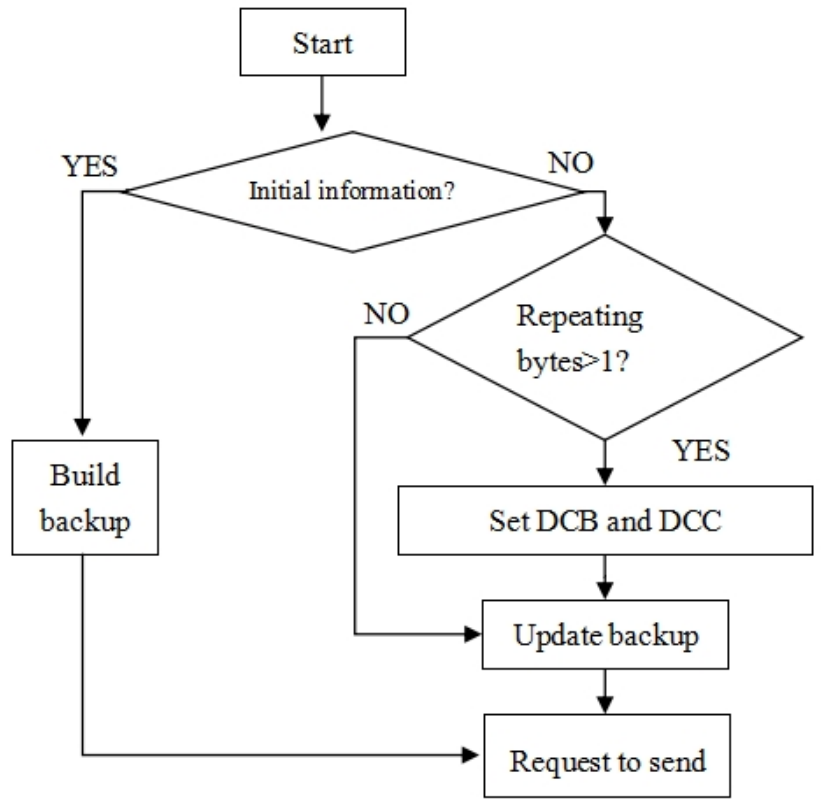

Fig.4 Flow chart of data compression in MDR

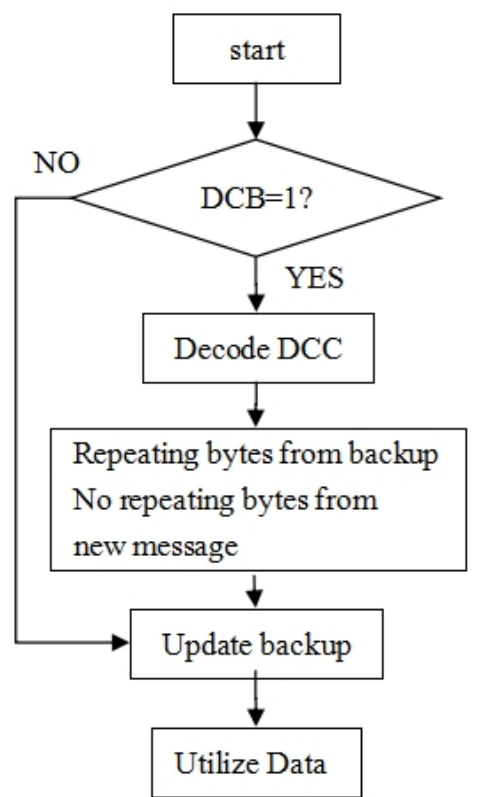

Fig.5 Flow chart of data decompression in MDR 


\section{Simulation study with network message time delays}

To study the effectiveness of the proposed controller, the cosimulations are carried out in Matlab/Simulink with a full vehicle model constructed by CarSim. The proposed algorithm is used in motion controller unit and a simple torque distribution strategy, which distributes the direct yawmoment equally to the driving or braking torques of 4 wheels, is used in torque distribution.

Fig. 5 shows the simulation results in single lane-changing maneuver. It is obvious in Fig. 5(a) and (b) that with conventional scheduling, the vehicle motion cannot track the desired reference and becomes unstable due to the network-induced time delays. While for controllers with MDR scheduling procedure, the adverse impact of delays can be eliminated and the control performance is satisfactory.

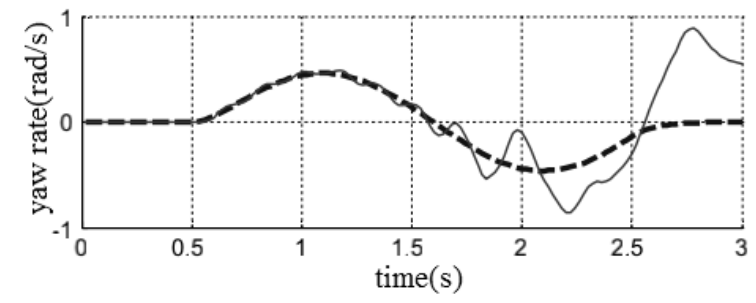

(a) Vehicle yaw rate with conventional controlling

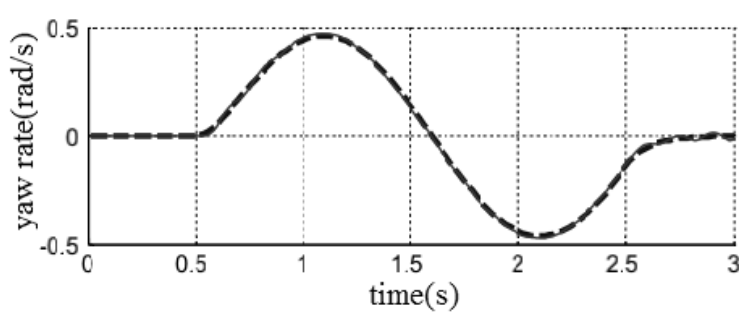

(b) Vehicle yaw rate with MDR scheduling

Fig.5 Vehicle yaw rate with conventional controlling and MDR scheduling (dashed-desired yaw rate, solid-yaw rate with control)

\section{Conclusions}

In this paper, the network-induced delays influence was discussed in 4WID-EV networked control system under direct yaw-moment control, to reduce the adverse impact of message timedelays, an alternative option is select to reduce the data transforming in network, a mended datareduction algorithm by compressing the four motor control messages into one message is proposed, the result of simulation with Simulink and CarSim demonstrate the effectiveness of the proposed algorithm, with MDR scheduling procedure, the adverse impact of delays can be eliminated and the control performance is satisfactory.

\section{Acknowledgements}

This research was financially supported by the National Natural Science Foundation of China (51405213, 51305181).

\section{References}

[1] A. Goodarzi and E. Esmailzadeh, Design of a VDC system for all-wheel independent drive vehicles, IEEE/ASME Transactions on Mechatronics, vol. 12, no. 6, pp. 632-639, 2007.

[2] Z. Shuai, H. Zhang, J. Wang, J. Li, and M. Ouyang, Combined AFS and DYC control of fourwheel-independent-drive electric vehicles over CAN Network with time-varying delays, IEEE Transactions on Vehicular Technology, vol. 63, no. 2, pp. 591-602, 2014.

[3] Y. H. Shen, Y. Gao, and T. Xu, Multi-axle vehicle dynamics stability control algorithm with all independent drive wheel, International Journal of Automotive Technology, vol. 17, no. 5, pp.795805, 2016.

[4] L. De Novellis, A. Sorniotti, P. Gruber et al. Direct yaw moment control actuated through electric drivetrains and friction brakes: theoretical design and experimental assessment, Mechatronics, vol. 26, pp. 1-15, 2015. 
[5] P. Song, C.F. Zong, and M. Tomizuka, A terminal sliding mode based torque distribution control for an individual-wheel-drive vehicle, Journal of Zhejiang University: Science A, vol. 15, no. 9, pp. 681-693, 2014.

[6]W.K Cao, Z.Y Liu, Y.H Chang et al, Direct Yaw-Moment Control of All-Wheel-IndependentDrive Electric Vehicles with Network-Induced Delays through Parameter-Dependent Fuzzy SMC Approach, Mathematical Problems in Engineering, Volume 2017, Article ID 5170492.

[7] Z. Shuai, H. Zhang, J. Wang, J. Li, and M. Ouyang, Lateral motion control for four-wheelindependent-drive electric vehicles using optimal torque allocation and dynamic message priority scheduling, Control Engineering Practice, vol. 24, no. 1, pp. 55-66, 2014.

[8] X. Zhu, H. Zhang, J. Wang, and Z. Fang, Robust lateral motion control of electric ground vehicles with random network-induced delays, IEEE Transactions on Vehicular Technology, vol.64, no. 11, pp. 4985-4995, 2015.

[9] Misbahuddin S, Mahmud S M, and Nizar A H. Development and performance analysis of a datareduction algorithm for automotive multiplexing, IEEE Transactions on Vehicular Technology. 2001, 50(1): 162-169. 